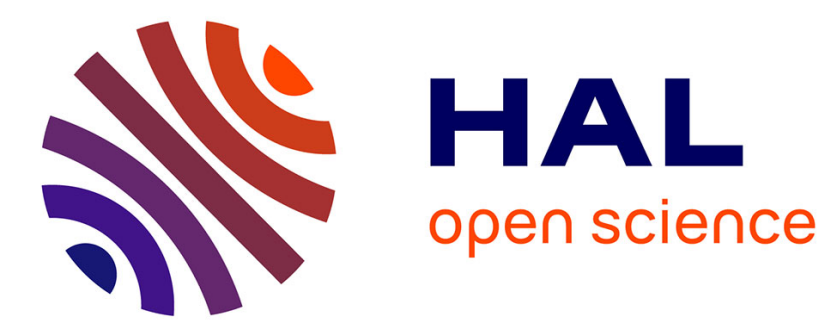

\title{
Robust Design of Parameter Identification
}

\author{
Aurélien Massein, David Daney, Yves Papegay
}

\section{To cite this version:}

Aurélien Massein, David Daney, Yves Papegay. Robust Design of Parameter Identification. ARK 2016

- Advances in Robotics Kinematics, Jun 2016, Grasse, France. hal-01405825

\section{HAL Id: hal-01405825 \\ https://hal.science/hal-01405825}

Submitted on 30 Nov 2016

HAL is a multi-disciplinary open access archive for the deposit and dissemination of scientific research documents, whether they are published or not. The documents may come from teaching and research institutions in France or abroad, or from public or private research centers.
L'archive ouverte pluridisciplinaire HAL, est destinée au dépôt et à la diffusion de documents scientifiques de niveau recherche, publiés ou non, émanant des établissements d'enseignement et de recherche français ou étrangers, des laboratoires publics ou privés. 


\title{
Robust Design of Parameter Identification
}

\author{
Aurélien Massein, David Daney and Yves Papegay
}

\begin{abstract}
Quality of results computed during parameter identification problems relies on the selection of system's states while performing measurements. This choice usually does not take into account the uncertainty of states and of measures. For identifiability, classical methods focus only on the contribution of model errors on the uncertainty of parameters. We present an alternative approach that tackles this drawback: taking into account influence of all uncertainty sources in order to improve parameter identification robustness to uncertainties. A robotic application example that showcases the differences between approaches is developed as well.
\end{abstract}

Key words: parameter identification, optimal design, observability index, uncertainties, localisation.

\section{Introduction}

Design of experiments [2] is a way to improve results of a generic parameter identification problem. It is applied namely in robotics [8], for calibration [1], and in GPS area through dilution of precision [9].

In such identification problem, unknown parameters are related with states of the system, and with measured outputs through a model prone to uncertainties. Inaccuracy sources of the model are model discrepancy, measurement errors and inexactitude of system's states. Observation of different sets of measurements for different states of the system provides a way to compute unknown parameters through

Aurélien Massein

Inria Bordeaux - Sud-Ouest, France e-mail:aurelien.massein@inria.fr

David Daney

Inria Bordeaux - Sud-Ouest, France e-mail:david.daneyeinria.fr

Yves Papegay

Inria Sophia Antipolis Méditerranée, France e-mail: yves .papegay@inria.fr 
a regression analysis process. In this process choice of states is crucial to enhance parameters identification. It refers to identifiability of the model and identifiability criteria.

In the non-linear case [5], unknown parameters are classically computed by an iterative optimisation algorithm, starting from an initial estimation. During this iterative process, states and measures remain unchanged and influence of their uncertainties are neglected. At the end of the process, all uncertainties have the same order of magnitude. Despite this last remark, in the literature, choice of states is done with the same assumption that uncertainties on states and measures are negligible, by evaluating identifiability criteria to the so-called identification matrix. In this paper, we promote the idea to take into account the different sources of inaccuracy -namely all uncertainty sources- when selecting states for parameter identification. Hence, we are applying similarly identifiability criteria to what we call the uncertainty matrix, a matrix describing the contribution of all uncertainties to errors of the model.

In the next section, we are describing with more details the parameter identification problem, the regression analysis process, the iterative optimisation process, and identifiability criteria. We carefully define the identification matrix and the uncertainty matrix, and how they are used for selection of identification states of the system. The last section is devoted to a pedagogical application, that clearly shows the difference between the classical and the proposed approach of states selection.

This application concerns the localisation of a source by a mobile robot. In this application, measures are taken at regular time step, and the selection of states correspond to a trajectory determination, that can be easily visualized. Another interest of this example is the ability to perform the states selection incrementally, that allows some enhancements of the identification method.

\section{Parameter Identification}

\subsection{Model Definition}

We consider a system in step $k=1 \ldots K$, depending on unknown parameters $x$, system's states $u=\left[\begin{array}{lll}u_{1} & \ldots & u_{k}\end{array}\right]^{T}$, and measured outputs $m=\left[\begin{array}{lll}m_{1} & \ldots & m_{k}\end{array}\right]^{T}$, through the following model:

$$
f_{k}\left(u_{k}, x, m_{k}\right)=0
$$

As we consider that system's states and measured outputs are prone to uncertainties, we distinguish each variable $x$ of its actual value $x^{*}$, and denote uncertainty on it by $\Delta x$. Once plugged into the model, the $k$-th observation of the system provides a set of $\operatorname{Dim}(f)$ equations:

$$
f_{k}\left(u_{k}^{*}, x^{*}, m_{k}^{*}\right)=f_{k}\left(u_{k}+\Delta u_{k}, x^{*}, m_{k}+\Delta m_{k}\right)=0
$$


We need to acquire $p$ system observations, such that the number of equations will be greater than the number of unknown parameters $(K \cdot \operatorname{Dim}(f)>\operatorname{Dim}(x))$. The resulting system is usually widely over-constrained.

\subsection{Regression Analysis}

Based on the set of these observations leading to residual errors,

$$
f_{k}\left(u_{k}, x^{*}, m_{k}\right)=\varepsilon_{k} \quad \text { with } \quad k=1 \ldots K
$$

a regression analysis estimator provides a solution $\hat{x}$ minimizing a given criterion. In the classical case of a Non-Linear Least Squares estimator, the criterion is the sum of the squares of residual errors:

$$
\sum_{k=1}^{K} f_{k}\left(u_{k}, \hat{x}, m_{k}\right)^{T} \cdot f_{k}\left(u_{k}, \hat{x}, m_{k}\right)
$$

Method of optimisation is based on a first-order linear approximation of the model:

$$
\begin{gathered}
f(u+\Delta u, x+\Delta x, m+\Delta m) \approx f(u, x, m)+J_{u} \cdot \Delta u+J_{x} \cdot \Delta x+J_{m} \cdot \Delta m \\
\quad \text { or } \\
\Delta f \approx J_{u} \cdot \Delta u+J_{x} \cdot \Delta x+J_{m} \cdot \Delta m
\end{gathered}
$$

with $\Delta u=\left[\Delta u_{1}, \ldots, \Delta u_{p}\right]^{T}, \Delta m=\left[\Delta m_{1}, \ldots, \Delta m_{p}\right]^{T}$ and $\Delta f=\left[\Delta f_{1}, \ldots, \Delta f_{p}\right]^{T}$.

Starting from an initial estimate $x_{0}$ of $x$, the Non-Linear Least Squares method performs several solving steps to reduce $\Delta x$. As $J_{u} \cdot \Delta u+J_{m} \cdot \Delta m$ is assumed to be negligible compared to $J_{x} \cdot \Delta x$, see [4], $j$-th step consists in solving the following linear system -with $\Delta f$ and $J_{x}$ computed from previous estimation $x_{j}$ - in the unknown variables $x_{j+1}$ :

$$
\Delta f\left[x_{j}\right]=J_{x}\left[x_{j}\right] \cdot \Delta x_{j+1} \quad \text { with } \quad \Delta x_{j+1}=x_{j+1}-x_{j}
$$

Iterative process ends when $\Delta x$ is sufficiently small, and is the same order of magnitude of $\Delta u$ and $\Delta m$ - see [4] for the stop condition.

At the end of this iterative process, one classically considers that the quality of the final estimation $\hat{x}$ relies on the numerical quality of the Jacobian matrix $J_{x}$, denoted by Identification Matrix. Numerical quality is precisely defined in terms of identifiability criteria (see below). As identification matrix depends on system's states, poses of the system for measurements are selected by optimisation of these criteria. 


\subsection{Identifiability Criteria}

Identifiability criteria have been widely studied in optimal design of experiments -being called optimality criteria- and in robotics calibration problems -being called observability criteria-. Sun and Hollerbach have synthesized in [10] those criteria and showed their similarities as some observability indexes have an optimality criterion counterpart.

Given a rectangular matrix $M$ such that $Y=M \cdot X$, identifiability criteria quantifies how uncertainties on $X$ propagate to uncertainties on $Y$ through $M$. The objective is to minimize some observability -or optimality- indexes, denoted $O$, associated to $M$, by choosing the state variable $\hat{u}$ which parametrize the matrix $M$, such that:

$$
\hat{u}=\underset{u}{\arg \min } O(M(u))
$$

Three criteria are popular, related to the singular values of the matrix $M$, obtained and sorted by a Singular Value Decomposition (and denoted by $\sigma_{L} \leq \ldots \leq \sigma_{1}$ ):

- D-Optimality: $O_{1}=\prod_{l=1}^{L} \sigma_{l}{ }^{1 /(n p)}$. This index corresponds to the determinant of $M^{T} . M$ here, $\sqrt{\operatorname{det}\left(M^{T} \cdot M\right)}=\prod_{l=1}^{L} \sigma_{l}$. The sensitivity of $Y$ with respect to $X$ is decreased when $O_{1}$ is minimized.

- Inverse of the condition number: $O_{2}=\sigma_{L} / \sigma_{1}$

- E-Optimality: $O_{3}=\sigma_{L}$

Sun and Hollerbach argued that $O_{1}$ (D-optimality) is the best criterion for a parameter estimation of an unscaled model or a model without a convincing scaling approach has to be minimized [10]. Fedorov and Leonov stated that D-optimal $\left(O_{1}\right)$ designs are most popular among theoretical and applied researchers in optimal experimental designs [2].

We can denote briefly that $\mathrm{O}_{2}$ which related to condition number is for balancing parameter uncertainties importance, whereas $\mathrm{O}_{3}$ is for reducing the worst parameter's uncertainty.

\subsection{Uncertainty Matrix}

At the end of the iterative process, a linear approximation of $f$ in the neighborhood of the final estimation $\hat{x}$ of $x$ is given by the $p$ following equations:

$$
f_{k}\left(u_{k}^{*}, x^{*}, m_{k}^{*}\right) \approx f\left(u_{k}, \hat{x}, m\right)+J_{u} \cdot \Delta u_{k}+J_{x} \cdot \Delta x+J_{m} \cdot \Delta m_{k}
$$

that can be written synthetically :

$$
\Delta f \approx J_{u} \cdot \Delta u+J_{x} \cdot \Delta x+J_{m} \cdot \Delta m
$$

or by introducing what we denote by $U$, the Uncertainty Matrix such that: 


$$
\Delta x=-J_{x}^{+} \cdot\left[\begin{array}{lll}
J_{u} & J_{m} & -I d
\end{array}\right] \cdot\left[\begin{array}{c}
\Delta u \\
\Delta m \\
\Delta f
\end{array}\right]=U \cdot\left[\begin{array}{c}
\Delta u \\
\Delta m \\
\Delta f
\end{array}\right]
$$

$J_{x}^{+}$being the pseudo-inverse of $J_{x}$.

We then claim that it is interesting to apply identifiability criteria to this uncertainty matrix $U$-minimizing $O(U)$ - when selecting states. To be efficient, at this point, we would benefit of normalizing the uncertainties as done in [6.7] or alternatively [3].

\section{Source Localisation Application}

In this section, we illustrate the difference between using the identifiability matrix and using the uncertainty matrix in the previously described states selection process, on a pedagogical two-dimensional source localisation application.

\subsection{Problem Statement}

We aim to localize accurately and step-efficiently a fixed source with the help of a mobile robot.

The mobile robot we consider has an embedded sensor measuring its direction with respect to a source $x=\left[\begin{array}{ll}x_{S} & y_{S}\end{array}\right]^{T}$, with a fixed sampling frequency and prone to bounded uncertainties. Our workspace is two dimensional and free of obstacle: wherever we are we can get a measure at each sampling step $k$ and move anywhere. Speed of the robot is supposed constant and a constant distance $r$ separates two consecutive measurement positions.

We describe the robot motion in polar coordinates. To move robot from position $u_{k}=\left[\begin{array}{ll}x_{k} & y_{k}\end{array}\right]^{T}$ at step $k$ onto the next position $u_{k+1}$, we need a leading direction $\alpha_{k+1}$ as written belowf:

$$
u_{k+1}=r\left[\begin{array}{c}
\cos \alpha_{k+1} \\
\sin \alpha_{k+1}
\end{array}\right]+u_{k}
$$

At each measurement step, the goniometric sensor on the robot provides the azimuth $m_{k}$-with respect to a fixed reference frame- such that

$$
\left[\begin{array}{c}
\sin m_{k} \\
-\cos m_{k}
\end{array}\right] \cdot\left(x-u_{k}\right)=0
$$

In the equations of the model, the position of the source $x$, the position $u_{k}$ and the measured azimuth $m_{k}$ play respectively the roles of unknown parameters $x$, states $u$ 
and measures $m$. Expressed at the $k$-th step, the model $f_{k}$ is precisely given by the previous equations. Please note that $u_{k}$ depends on $\alpha_{k}$.

Hence first-order linear approximation of the model expressed as:

$$
\left[\begin{array}{c}
\sin m_{k} \\
-\cos m_{k}
\end{array}\right] \cdot \Delta x+\left[\begin{array}{c}
-\sin m_{k} \\
\cos m_{k}
\end{array}\right] \cdot \Delta u_{k}+\left[\begin{array}{c}
\cos m_{k} \\
-\sin m_{k}
\end{array}\right] \cdot\left(x-u_{k}\right) \cdot \Delta m_{k}
$$

The difference between robot and source position $x-u_{k}$ is equal to $\rho_{k}$, the distance between $u_{k}$ and $x$, modulo $\cos \left(m_{k}^{*}-m_{k}\right)$ which approximation is considered equal to 1 .

\subsection{States Choices}

The expressions of the identifiability matrix and of the uncertainty matrix are respectively (at the $k$-th step):

$$
\begin{aligned}
& J_{x_{k}}=\left[\begin{array}{c}
j x_{1} \\
\ldots \\
j x_{k}
\end{array}\right] \quad \text { with } \quad j x_{k}=\left[\sin m_{k},-\cos m_{k}\right] \\
& U_{k}=J_{x_{k}}^{+} \cdot N_{k} \\
& \text { where } N_{k}=\left[\begin{array}{cccc}
n_{1} & 0 & \ldots & 0 \\
0 & n_{2} & 0 & \vdots \\
\vdots & 0 & \ddots & 0 \\
0 & \ldots & 0 & n_{k}
\end{array}\right] \quad \text { with } n_{k}=\left[\begin{array}{lll}
\sin m_{k}-\cos m_{k} & -\rho_{k} & 1
\end{array}\right]
\end{aligned}
$$

Then, the optimal next direction $\alpha_{k+1}$ for the mobile robot, is defined with the help of the identifiability criterion $O_{1}$ using either $J_{x_{k+1}}$ or $U_{k+1}$.

\subsection{Experiment and Results}

In our experiment, source $x$ is at position $x=\left[\begin{array}{ll}0 & 1\end{array}\right]^{T}$, and the initial mobile robot position is $u_{1}=\left[\begin{array}{ll}0 & 0\end{array}\right]^{T}$. The fixed motion step is $r=0.01$, with a relative error lower than $1 \%$ and we have the same error on the direction $\alpha_{k}$ about $0.1^{\circ}$. Finally, the uncertainty of the measured angle $\Delta m$ is uniformly distributed and bounded by $\Delta m= \pm 10^{\circ}$.

In a first experiment, we select each step $\alpha_{k+1}$ according to the maximization of $O_{1}\left(J_{x_{k+1}}\right)$. In the second, we select it to minimize $O_{1}\left(U_{k+1}\right)$.

The obtained results are presented in figures $1 \mathrm{a}$ ) and $1 \mathrm{~b}$. 
The first one displays the motion of the mobile robot into the euclidean workspace step-by-step: blue motion curve stands for a gradient determinant optimisation on identification matrix $J_{x}$ and orange motion curve for one on uncertainty matrix $U$.

The second one presents the error $\left\|x^{*}-\hat{x}\right\|$ as a function of the number of steps in the two experiments with the same color code.

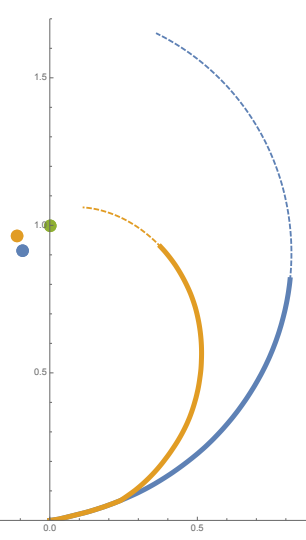

(a) Robot motions

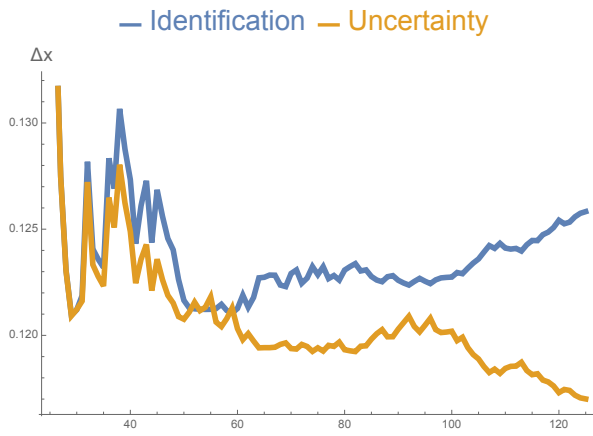

(b) Parameters Difference

Fig. 1: Source Localisation Application

Note that the blue motion favours a circle approach centered on the source position whereas the orange motion favours a spiral approach on it: By neglecting uncertainties on measures (blue case), we intuitively want to change the angle $m$ as much as possible between two measurement positions, see [11], for a better conditioning of $J_{x_{k}}$. On the orange trajectory, we also take into account the measurement errors, so we try to become closer to the source to minimize the influence of such errors.

\section{Conclusion}

We have introduced a new matrix to improve parameter estimation robustness to all uncertainties, in complement to classical identification optimisation. We applied our matrix optimisation in a source localisation application and demonstrated its possible application in a practical case. Results outperformed classical identification optimisation.

This application highlights that, to improve parameter estimation accuracy, we need to take into account more uncertainties than classically ones held in the identification matrix. Our proposed uncertainty matrix can be used in design of parameter 
identification problems, can improve significantly estimations accuracy and their robustness to uncertainties.

\section{References}

1. Daney, D., Papegay, Y., Madeline, B.: Choosing Measurement Poses for Robot Calibration with the Local Convergence Method and Tabu Search. The International Journal of Robotics Research 24(6), 501-518 (2005). DOI 10.1177/0278364905053185. URL http://ijr. sagepub.com/content/24/6/501

2. Fedorov, V.V., Leonov, S.L.: Optimal Design for Nonlinear Response Models. CRC Press (2013)

3. Gayral, T.: Étalonnage d'un instrument d'observation spatial actif. phdthesis, Université Nice Sophia Antipolis (2013). URL https://tel.archives-ouvertes.fr/ tel-00950898/document

4. Gayral, T., Daney, D.: A sufficient condition for parameter identifiability in robotic calibration. In: F. Thomas, A. Perez Gracia (eds.) Computational Kinematics, Mechanisms and Machine Science, vol. 15, pp. 131-138. Springer Netherlands (2014). DOI 10.1007/978-94-007-7214-4_15. URL http://dx.doi.org/10.1007/ 978-94-007-7214-4_15

5. Hollerbach, J., Khalil, W., Gautier, M.: Model Identification. In: B.S. Prof, O.K. Prof (eds.) Springer Handbook of Robotics, pp. 321-344. Springer Berlin Heidelberg (2008). URL http://link.springer.com/referenceworkentry/10.1007/ 978-3-540-30301-5_15

6. Hollerbach, J.M.: Advances in robot calibration. In: In 6th Int. Symposium on Robotics Research, p. 319-326

7. Hollerbach, J.M., Wampler, C.W.: The calibration index and taxonomy for robot kinematic calibration methods. The international journal of robotics research 15(6), 573-591 (1996)

8. Kelly, A.: Precision dilution in triangulation based mobile robot position estimation. In: Intelligent Autonomous Systems, vol. 8, pp. 1046-1053 (2003). URL http://www. frc.ri. Cmu.edu/ alonzo/pubs/papers/ias8.pdf

9. Langley, R.B.: Dilution of precision. GPS world 10(5), 52-59 (1999). URL http://www.nrem.iastate.edu/class/assets/nrem446_546/week3/ Dilution_of_Precision.pdf

10. Sun, Y., Hollerbach, J.: Observability index selection for robot calibration. In: IEEE International Conference on Robotics and Automation, 2008. ICRA 2008, pp. 831-836 (2008). DOI 10.1109/ROBOT.2008.4543308

11. Takeda, Y., Shen, G., Funabashi, H.: A dbb-based kinematic calibration method for in-parallel actuated mechanisms using a fourier series. Journal of Mechanical Design 126(5), 856-865 (2004) 